\title{
Targeted disruption of the Chop gene delays endoplasmic reticulum stress-mediated diabetes
}

\author{
Seiichi Oyadomari, ${ }^{1,2}$ Akio Koizumi, ${ }^{3}$ Kiyoshi Takeda, ${ }^{4}$ Tomomi Gotoh, ${ }^{1}$ Shizuo Akira, ${ }^{4}$ \\ Eiichi Araki, ${ }^{2}$ and Masataka Mori ${ }^{1}$ \\ ${ }^{1}$ Department of Molecular Genetics, and \\ ${ }^{2}$ Department of Metabolic Medicine, Kumamoto University School of Medicine, Kumamoto, Japan \\ ${ }^{3}$ Department of Health and Environmental Sciences, Kyoto University School of Public Health, Kyoto, Japan \\ ${ }^{4}$ Department of Host Defense, Research Institute for Microbial Diseases, Osaka University and Japan Science \\ and Technology Corp., Osaka, Japan
}

Address correspondence to: Masataka Mori, Department of Molecular Genetics, Kumamoto University School of Medicine, Honjo 2-2-1, Kumamoto 860-0811, Japan.

Phone: 81-96-373-5140; Fax: 81-96-373-5145; E-mail: masa@gpo.kumamoto-u.ac.jp.

Received for publication November 1, 2001, and accepted in revised form December 28, 2001.

\begin{abstract}
Overload of pancreatic $\beta$ cells in conditions such as hyperglycemia, obesity, and long-term treatment with sulfonylureas leads to $\beta$ cell exhaustion and type 2 diabetes. Because $\beta$ cell mass declines under these conditions, apparently as a result of apoptosis, we speculated that overload kills $\beta$ cells as a result of endoplasmic reticulum (ER) stress. The Akita mouse, which carries a conformation-altering missense mutation (Cys96Tyr) in Insulin 2, likewise exhibits hyperglycemia and a reduced $\beta$ cell mass. In the development of diabetes in Akita mice, mRNAs for the ER chaperone Bip and the ER stress-associated apoptosis factor Chop were induced in the pancreas. Overexpression of the mutant insulin in mouse MIN6 $\beta$ cells induced Chop expression and led to apoptosis. Targeted disruption of the Chop gene delayed the onset of diabetes in heterozygous Akita mice by $8-10$ weeks. We conclude that ER overload in $\beta$ cells causes ER stress and leads to apoptosis via Chop induction. Our findings suggest a new therapeutic approach for preventing the onset of diabetes by inhibiting Chop induction or by increasing chaperone capacity in the ER.
\end{abstract}

J. Clin. Invest. 109:525-532 (2002). DOI:10.1172/JCI200214550.

\section{Introduction}

Type 2 diabetes is characterized by insulin resistance and pancreatic $\beta$ cell dysfunction. Hyperglycemia occurs by a progressive failure of pancreatic $\beta$ cells to secrete sufficient amounts of insulin to compensate for the insulin resistance. Thus, dysfunction of $\beta$ cell secretion is a key element in the pathophysiology of type 2 diabetes. Increased demand for insulin secretion under certain conditions, such as obesity and long-term treatment with sulfonylureas, may result in $\beta$ cell overload and insulin secretion deficiency. $\beta$ cell dysfunction under these conditions is often termed "pancreatic $\beta$ cell exhaustion." Several studies have shown that $\beta$ cell mass (both number and individual volume of cells) is reduced in patients with type 2 diabetes $(1,2)$, an event possibly due to apoptotic death of $\beta$ cells and to a reduction of cell proliferation. Precise mechanisms remain to be determined.

One of the characteristic features of $\beta$ cells is a highly developed endoplasmic reticulum (ER), apparently due to heavy engagement in insulin secretion. The ER serves several important functions, including posttranslational modification, folding, and assembly of newly synthesized secretory proteins, and its proper function is essential to cell survival. The ER is regulated by signaling pathways that respond to an accumulation of unfolded or misfolded proteins in the organelle. At least four functionally distinct responses have been identified (3-5). The first response involves upregulation of genes encoding ER chaperone proteins, such as Bip/GRP78 and GRP94, to increase protein folding activity and prevent protein aggregation. The second response consists of translational attenuation, to reduce the load of new protein synthesis and prevent further accumulation of unfolded proteins. The third is degradation of misfolded proteins in the ER, a process known as ER-associated degradation. The misfolded proteins are transported from the ER to the cytosol, where ubiquitin-conjugating enzymes target them for degradation by the $26 \mathrm{~S}$ proteasome. The last response is apoptosis, which occurs when functions of the ER are extensively impaired. This apoptotic event is mediated by transcriptional activation of the gene for Chop (C/EBP homologus protein, also known as GADD153), a member of the C/EBP family of transcription factors $(6,7)$, and by activation of ER-associated caspase-12 (8). The upstream components of these response pathways consist of several transmembrane proteins in the ER that transmit stress signals in response to perturbation of protein folding. ER stress transducer proteins, including Ire $1 \alpha$ and PERK, are expressed at high levels in $\beta$ cells. Mice lacking PERK were found to exhibit $\beta$ cell 
death and to develop diabetes (9). Mice with a homozygous mutation at the eIF $2 \alpha$ phosphorylation site have a $\beta$ cell deficiency (10). These data suggest that $\beta$ cells are affected by ER stress under conditions lacking any sign of insulin resistance (such as obesity). Moreover, we found that nitric oxide-induced apoptosis in $\beta$ cells is mediated by the ER-stress pathway (11). Nitric oxide depletes $\mathrm{ER} \mathrm{Ca}^{2+}$, causes ER stress, and leads to apoptosis through Chop induction. Pancreatic islets from Chop knockout mice showed resistance to nitric oxide. High expression of these ER stress transducer proteins may be necessary for strict quality control of secretory proteins in $\beta$ cells, and the disturbance in protein traffic in the ER may lead to cell death.

A mutant mouse line, called the Akita mouse, which spontaneously develops hyperglycemia with reduced $\beta$ cell mass, but without insulitis or obesity, was established $(12,13)$. Genetic analysis revealed that a mutation of the insulin 2 gene (Ins2) (Cys96Tyr) is responsible for the diabetic phenotype in this mouse (14). This mutation disrupts a disulfide bond in insulin between the A and B chains and is thought to induce a drastic conformational change of this molecule. We speculated that this mouse could serve as a diabetic model related to ER stress. We now report that progressive hyperglycemia in the Akita mouse is accompanied by Chop induction and $\beta$ cell apoptosis. Targeted disruption in the Chop gene delayed the onset of disease in heterozygous Akita mice. These results highlight the importance of chronic ER stress in $\beta$ cell apoptosis and in the reduced islet mass observed in type 2 diabetes.

\section{Methods}

Generation of animal models. All procedures involving these animals were approved by the Animal Care and Use Committee of Kumamoto University. Mice lacking the Chop gene (C57BL/6 background) were generated as described (11). Akita mice, which carry the Cys96Tyr (C96Y) missense mutation in the Ins2 gene, were established on a $\mathrm{C} 57 \mathrm{BL} / 6$ background and characterized as described $(12,13)$. Heterozygous mutants for Ins2 (Ins2 ${ }^{W T / C 96 Y)}$ were crossed with homozygous mutants for the Chop gene (Chop $\left.{ }^{--}\right)$, generating Ins2 ${ }^{W T / W T} \mathrm{Chop}^{+/-}$and Ins2 ${ }^{W T / C 96 Y} \mathrm{Chop}^{+/-}$mice. Ins2 ${ }^{\text {WT/C96Y }} \mathrm{Chop}^{+/-}$mice were crossed with Ins2 ${ }^{\text {WT/C96Y }} \mathrm{Chop}^{+/-}$or Ins2 ${ }^{\text {WT/WT }} \mathrm{Chop}^{-/-}$mice, which yielded nine genotypes of mice: Ins $2^{W T / W T} \mathrm{Chop}^{+/+}$, Ins2 ${ }^{\text {WT/WT }} \mathrm{Chop}^{+/-}$, Ins2 ${ }^{W T / W T} \mathrm{Chop}^{-/-}$, Ins2 ${ }^{\mathrm{WT} /{ }^{\mathrm{C}} 96 \mathrm{CY}} \mathrm{Chop}^{+/+}$, Ins2 ${ }^{\mathrm{WT} / \mathrm{C} 96 \mathrm{C}} \mathrm{Chop}^{+/-}$, Ins2 ${ }^{\mathrm{WT} / \mathrm{C} 96 \mathrm{C}} \mathrm{Chop}^{-/-}$, Ins2 ${ }^{\mathrm{C} 96 \mathrm{Y} / \mathrm{C} 96 \mathrm{C}} \mathrm{Chop}^{+/+}$, Ins $2^{\mathrm{C} 96 \mathrm{Y} / \mathrm{C} 96 \mathrm{C}} \mathrm{Chop}^{+/-}$, and Ins $2^{\mathrm{C} 96 \mathrm{Y} / \mathrm{C} 96 \mathrm{Y}} \mathrm{Chop}^{-/-}$.

Genotyping of the double-mutant mice. The genotype for Ins 2 was determined by RFLP, as described (14). The genotype for Chop was determined by PCR. The following primers were used: the sense primer was $5^{\prime}$ GAGAAAAAAAGAGTACAAATGGCCTGG- ${ }^{\prime}$, and the antisense primers were $5^{\prime}$-AATTTCATCTGAGGACAGGACCTCCTG-3' (derived from the neomycin resistance gene), and $5^{\prime}$-ATCGCCTTCTATCGCCTTCTTGACGAG-3' (derived from the Chop gene). The thermal cycle reaction was performed as follows: $94^{\circ} \mathrm{C}$ for 2 minutes, followed by 35 cycles at $94^{\circ} \mathrm{C}$ for 15 seconds, $55^{\circ} \mathrm{C}$ for 30 seconds, $68^{\circ} \mathrm{C}$ for 2 minutes, and $72^{\circ} \mathrm{C}$ for 7 minutes. The wild-type and recombinant alleles each yielded a transcript of $1.3 \mathrm{~kb}$.

Northern blot analysis. Total RNA ( $2.0 \mu \mathrm{g}$ per lane) was electrophoresed in formaldehyde-agarose gels, and blotted onto nylon membranes. Northern blot analysis was done as described (15). Digoxigenin-labeled antisense RNA probes for mouse insulin 2 (nucleotides 2344-3026; GenBank accession number NM_008387), mouse Chop (11), and mouse Bip (nucleotides 1629-1859; GenBank accession number AJ002387) were synthesized using a DIG RNA labeling kit (Roche Molecular Biochemicals, Indianapolis, Indiana, USA). Chemiluminescence signals were quantified using a chemiluminescence image analyzer (LAS-1000plus; Fuji Photo Film Co., Tokyo, Japan).

Concentrations of blood glucose and plasma insulin. Blood glucose concentration was measured by the mutarotase/glucose dehydrogenase method, using a glucose analyzer (HemoCue AB, Ängelholm, Sweden). Plasma insulin concentration was measured by a sandwichtype enzyme immunoassay using an insulin ELISA kit (Shibayagi Co., Shibukawa, Japan).

Insulin content in the pancreas. Pancreata were removed from mice and immediately frozen in liquid nitrogen. Protein extracts were prepared using the acid/ethanol method (16). Briefly, the tissue was homogenized in acid/ethanol ( $0.18 \mathrm{M} \mathrm{HCl}$ in $70 \%$ ethanol), and the homogenate was sonicated for 15 seconds and kept at $4^{\circ} \mathrm{C}$ for 24 hours. Homogenates were then centrifuged at $500 \mathrm{~g}$ for 10 minutes at $4^{\circ} \mathrm{C}$, and the supernatant was stored at $-20^{\circ} \mathrm{C}$. After neutralization, insulin in the extracts was measured using insulin ELISA as described above. Protein in tissue extracts was determined by the Bradford method.

Immunostaining and TUNEL assay. Pancreas specimens were fixed with buffered $4 \%$ paraformaldehyde solution, and embedded in paraffin. Blocks were cut into $5-\mu \mathrm{m}$-thick sections. Sections were stained with a rabbit anti-insulin polyclonal antibody (Santa Cruz Biotechnology Inc., Santa Cruz, California, USA) or a mouse antiChop monoclonal antibody (Santa Cruz Biotechnology Inc.) as the primary antibody; $\mathrm{Cy} 3$-labeled anti-rabbit IgG (Amersham Pharmacia Biotech, Piscataway, New Jersey, USA) or Alexa Fluor 488-labeled anti-mouse IgG (Molecular Probes Inc., Eugene, Oregon, USA) was used as the secondary antibody. For apoptosis detection, the TUNEL assay was performed using an in situ apoptosis detection kit (Takara Shuzo Co., Otsu, Japan). The tissues were viewed under an Olympus fluorescence microscope equipped with a krypton-argon laser, and images were acquired using a C5810 color chilled 3CCD video camera system (Hamamatsu Photonics KK, Hamamatsu, Japan).

Cell culture, transfection, and apoptosis analysis. Mouse insulinoma MIN6 cells were cultured in 60-mm collagen-coated dishes in DMEM supplemented with 25 $\mathrm{mM}$ glucose and 10\% FCS, as described (11). 
Plasmid DNAs ( $5 \mu \mathrm{g}$ in total) were transfected into MIN6 cells using LipofectAMINE 2000 (Invitrogen Corp., Carlsbad, California, USA). An expression vector for enhanced green fluorescent protein (pEGFP) and an ER localization vector that expresses enhanced yellow fluorescent protein (pEYFP-ER) were purchased from Clontech Laboratories Inc. (Emeryville, California, USA). The mouse insulin 2 cDNA clone was isolated by RT-PCR, using mRNA from MIN6 cells. To introduce the Cys96Tyr substitution, overlap extension PCR mutagenesis was done using the mutant primers $5^{\prime}$-AGCGTGGCATTGTAGATCAGTGCTACACCA- $3^{\prime}$ and 5'-TGGTGTAGCACTGATCTACAATGCCACGCT- 3 '. The mutation was confirmed by DNA sequencing. Construction of pcDNA-Ins2 ${ }^{\text {WT }}$ and pcDNA-Ins2 ${ }^{C 96 Y}$ was performed by inserting the full-length cDNA fragment for mouse wild-type or Cys96Tyr, mutant insulin 2 respectively, into the mammalian expression vector pcDNA3.1(-) (Invitrogen Corp.) after linker attachment. To generate the $\mathrm{pIns} 2^{\mathrm{WT}}$-EGFP and the $\mathrm{pIns} 2^{\mathrm{C} 96 \mathrm{Y}_{-}}$ EGFP constructs, in which EGFP was located at the C-terminus of insulin, the full-length cDNA fragment, full-length except for its termination codon, was inserted into the mammalian expression vector PEGFP-N1 (Clontech Laboratories Inc.).

For the apoptosis assay, 48 hours after transfection, the medium was carefully removed to collect detached cells. Adherent cells were recovered by trypsinization and mixed with the separated medium. Cells were pelleted and resuspended. To analyze apoptotic changes of nuclei, cells were fixed in $4 \%$ formaldehyde in PBS for 30 minutes, stained with Hoechst 33258, and observed under a fluorescence microscope. To detect DNA ladder formation, DNA was extracted from cells, electrophoresed in $2 \%$ agarose gel, stained with SYBR Green I (Molecular Probes Inc.), and visualized by UV transillumination. Annexin V staining was performed using the Annexin V-Cy3 apoptosis detection kit (Medical and Biological Laboratories Co., Nagoya, Japan) according to the manufacturer's instructions.

Statistical analysis. Data were expressed as mean \pm SD. Statistical significance of differences between groups was evaluated using the unpaired Student $t$ test. When the $P$ value was less than 0.05 , the difference was considered to be statistically significant.

\section{Results}

Diabetic phenotype of mice with Ins 2 mutation. To examine the phenotypic consequences of Ins 2 mutation in the Akita mouse, we monitored blood glucose levels in each genotype (Figure 1). Ins $2^{\mathrm{C} 96 \mathrm{Y} / \mathrm{C} 96 \mathrm{Y}}$ mice developed hyperglycemia at 2 weeks of age, and blood glucose levels continued to increase up to $700 \mathrm{mg} / \mathrm{dl}$ at 10 weeks of age. At 2 weeks of age, Ins2 ${ }^{W T / C 96 Y}$ mice had euglycemia. They developed diabetes between 4 and 8 weeks of age. Thus, the severity and the onset of disease correlated well with number of the mutant allele. All Akita mice developed diabetes (100\% penetration) and showed the simultaneous onset of disease in each genotype. There was no gender difference in onset of the disease. However, the increase in blood glucose levels was more marked in male than in female mice, both Ins2 ${ }^{\mathrm{C} 96 Y / C 96 Y}$ and Ins2 ${ }^{W T / C 96 Y}$.

Induction of ER stress-associated genes in the pancreas of Akita mice. The Ins2 mutation in Akita mice disrupts a disulfide bond between the $\mathrm{A}$ and $\mathrm{B}$ chains of insulin, and can induce a drastic conformational change in the molecule. We speculated that this misfolded insulin could cause ER stress. To check ER stress in the Akita mice, Northern blot analysis for insulin and ER stress-associated proteins in pancreas was done (Figure 2). In Ins $2^{W T / W T}$ mice, insulin mRNA gradually increased with age (data not shown). At 3 weeks of age, insulin mRNA was higher in Ins2 ${ }^{W T / C 96 Y}$ mice than in Ins2 ${ }^{W T / W T}$ mice, and was higher yet in Ins $2^{C 96 Y / C 96 Y}$ mice. At 6 weeks of age, insulin mRNA was higher in Ins $2^{W T / C 96 Y}$ mice than in controls, but was similar between Ins $2^{\text {WT/C96Y }}$ mice and Ins $2^{C 96 Y / C 96 Y}$ mice. In contrast, at 9 weeks of age, the mRNA was slightly higher in Ins2 ${ }^{W T / C 96 Y}$ mice than in controls, and was much lower in Ins $2^{\mathrm{C} 96 \mathrm{Y} / \mathrm{C} 96 \mathrm{Y}}$ mice than in controls. This decrease in insulin mRNA in Ins $2^{\mathrm{C} 96 Y / C 96 Y}$ mice appears to be due to a loss of $\beta$ cells (see below). Of note is that the onset of diabetes is
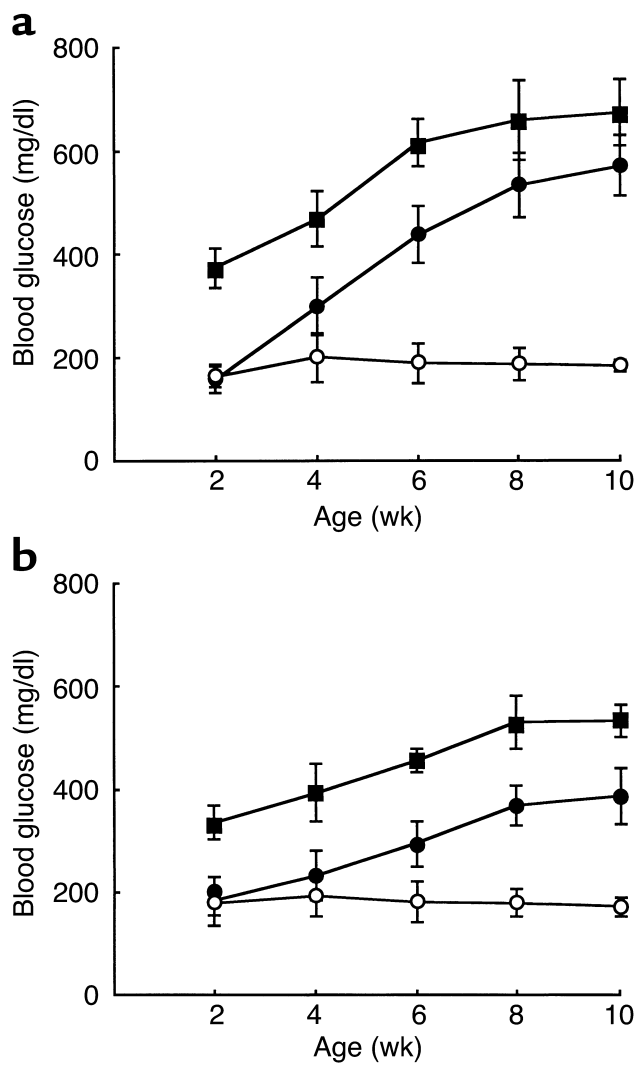

Figure 1

Development of diabetes in Akita mice. Mice were provided food ad libitum, and blood glucose was measured between 9:00 a.m. and 10:00 a.m. at indicated age. (a) Male. (b) Female. Wild-type Ins $2^{W T / W T}$ mice (open circles), heterozygous Ins $2^{W T / C 96 Y}$ mice (filled circles), and homozygous Ins2 ${ }^{\mathrm{C} 96 \mathrm{Y} / \mathrm{C} 96 \mathrm{Y}}$ mice (filled squares) are represented. Data are shown as mean \pm SD $(n=10)$. 


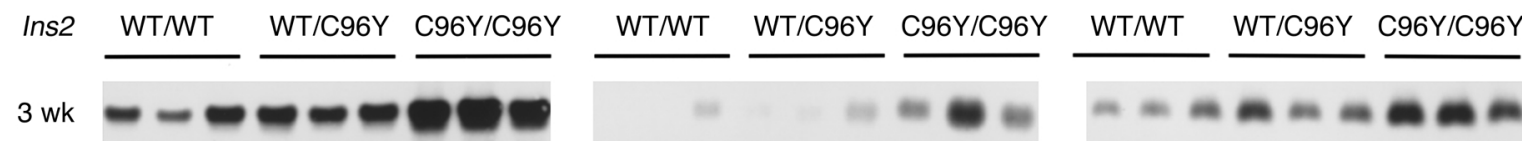

6 wk

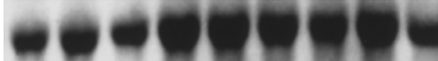

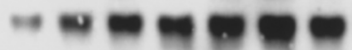

9 wk
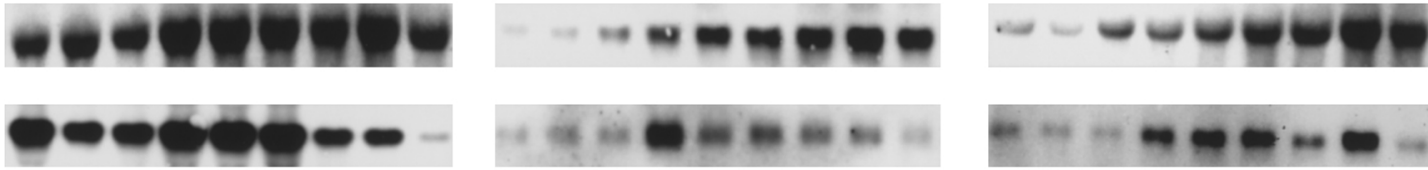

b
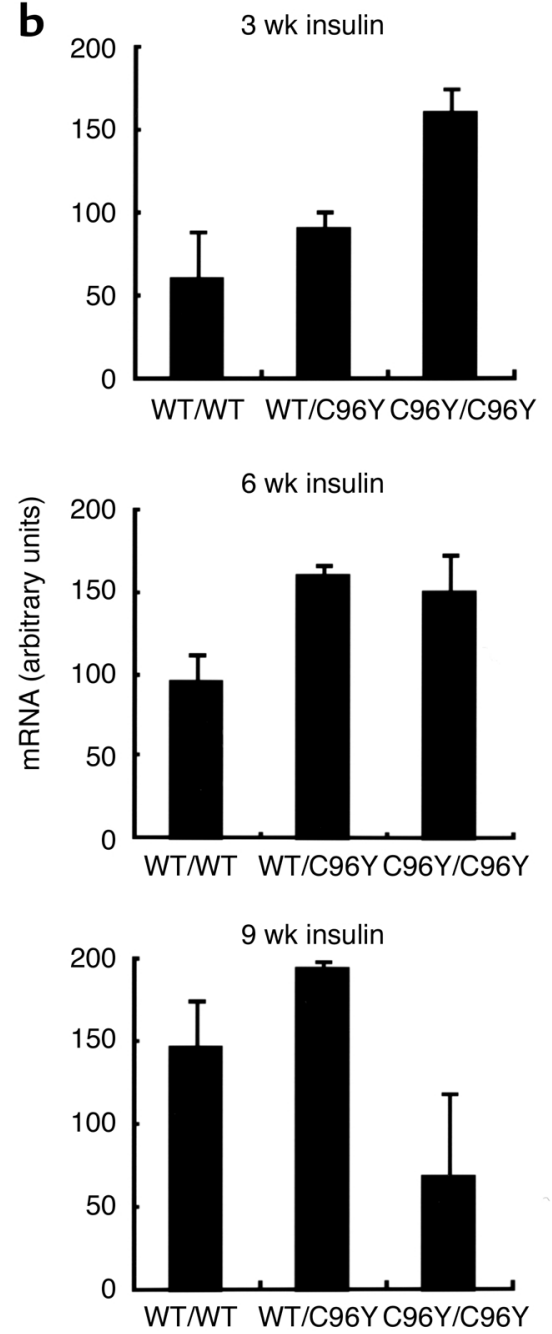
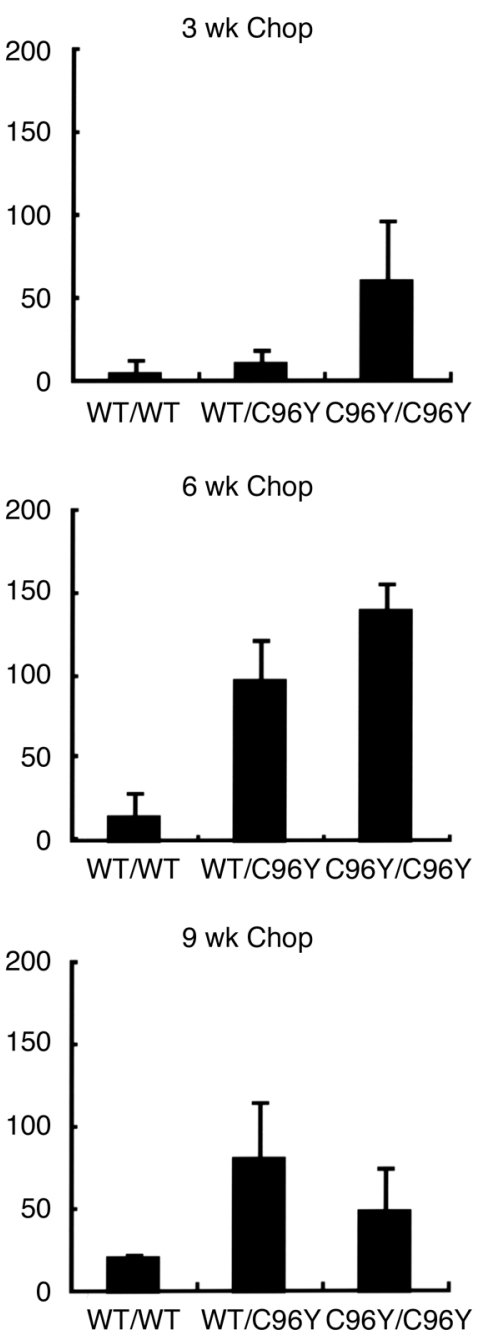
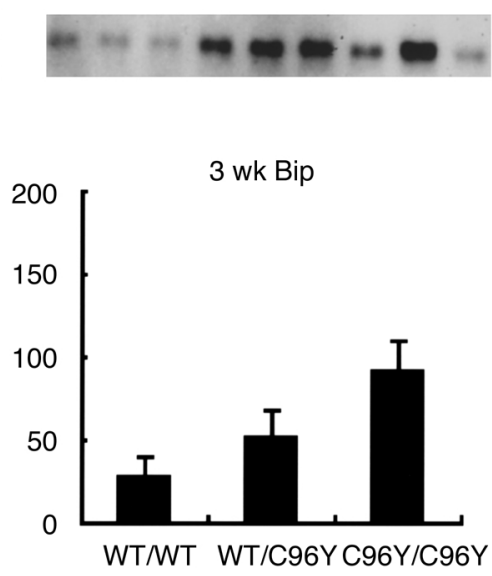

6 wk Bip
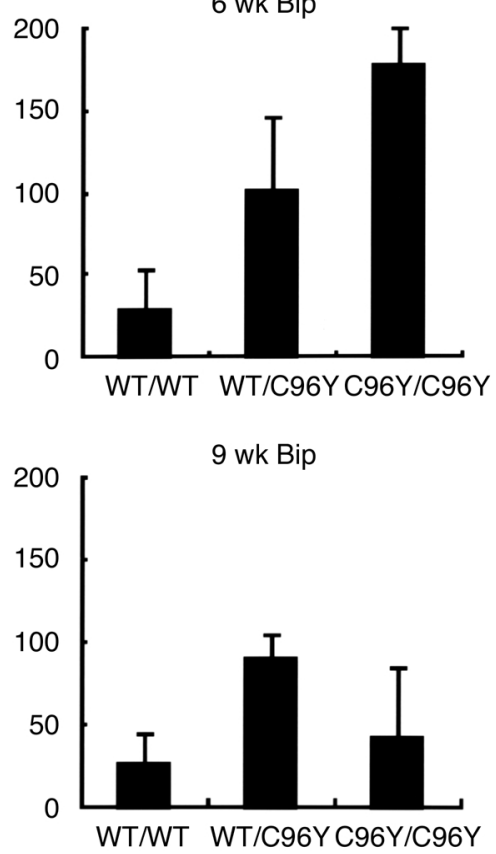

Figure 2

Northern blot analysis for insulin, Chop, and Bip mRNAs in the pancreas of male Akita mice. (a) Chemiluminescent images for insulin, Chop, and Bip mRNAs at the indicated ages are shown. Total RNAs $(2.0 \mathrm{mg})$ from the pancreas of each genotype were subjected to Northern blot analysis. The results at 3,6 , and 9 weeks of age were obtained under essentially identical conditions. (b) Quantification of the results obtained in a, shown as mean $\pm \mathrm{SD}(n=3)$.

associated with an increase in insulin mRNA. This suggests that insulin mRNA is induced to compensate for the deficiency in secretion of active insulin. Chop/GADD153, a transcription factor that plays a role in growth arrest and cell death, and Bip/GRP78, an ER chaperone, are induced by ER stress. Chop mRNA was much higher in Ins $2^{\mathrm{C} 96 Y / C 96 Y}$ mice than in controls at 3 weeks of age. It was also much higher than that in con- trols in both Ins2 ${ }^{2 T / C 96 Y}$ mice and Ins2 ${ }^{C 96 Y / C 96 Y}$ mice at 6 weeks of age, and in Ins $2^{W T / C 96 Y}$ mice at 9 weeks of age. The profile of fluctuation in Chop mRNA was similar to that of insulin mRNA. Bip mRNA and Chop mRNA changed in a similar manner. These results indicate that $\beta$ cells in both heterozygous and homozygous mutant mice are subjected to ER stress, and that the ER stress is closely associated with the induction of insulin mRNA. 


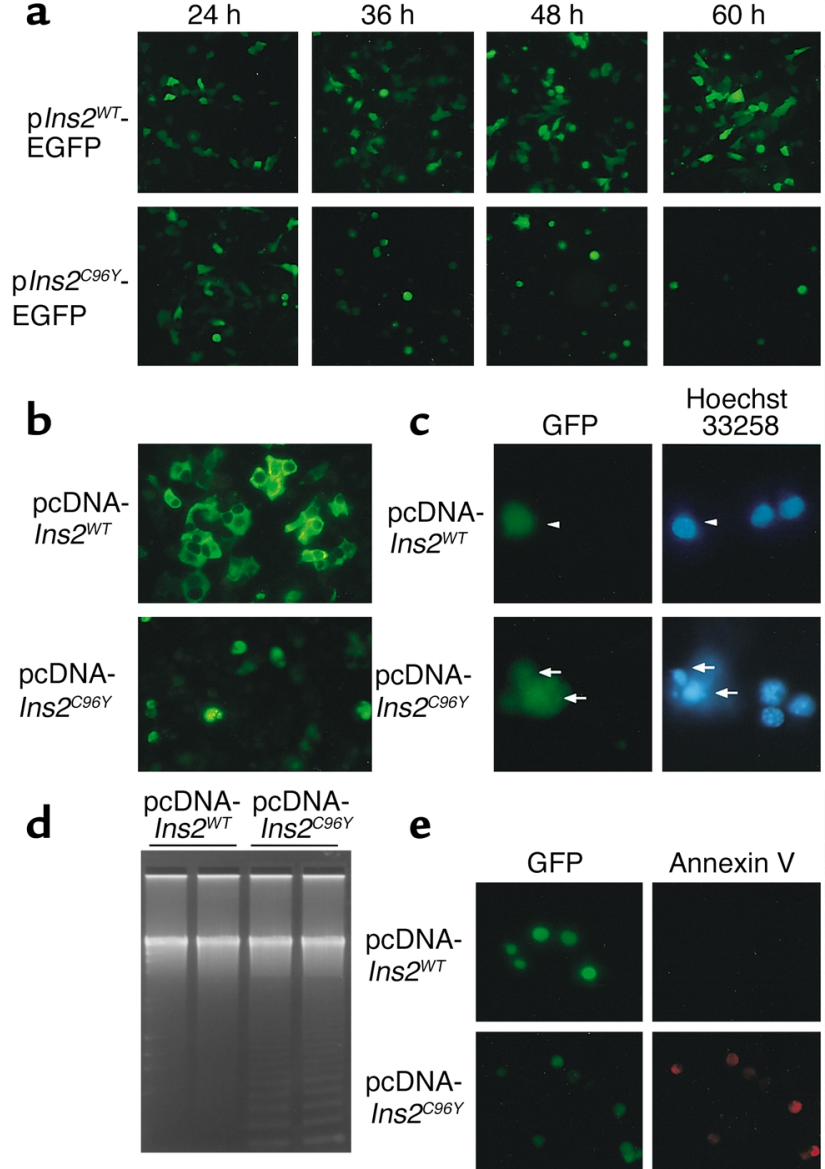

Figure 3

Apoptosis in MIN6 cells overexpressing Ins2 ${ }^{\mathrm{C} 96 \mathrm{Y}}$-EGFP and Ins2 ${ }^{\mathrm{C} 96 \mathrm{Y}}$. (a) Cells were transfected with p/ns2 $2^{W T}$-EGFP or plns2 ${ }^{\text {C96Y }}$-EGFP. At the indicated times after transfection, cells were observed under a fluorescence microscope. Original magnification: $\times 100$. (b) Cells were cotransfected with PEYFP-ER and either PcDNA-Ins $2^{W T}$ or pcDNA-Ins $2^{C 96 Y}$. Forty-eight hours after transfection, cells were observed under a fluorescence microscope. Original magnification: $\times 200$. (c) Cells were cotransfected with pEGFP and either pcDNAIns $2^{W T}$ or pcDNA-Ins $2^{C 96 Y}$. The transfected cells and apoptotic cells were visualized by GFP fluorescence and Hoechst 33258 staining, respectively. pcDNA-Ins $2^{W T}$-transfected cells were not apoptotic (arrowheads), whereas pcDNA-Ins $2^{C 96 Y}$-transfected cells were apoptotic (arrows). Original magnification: $\times 400$. (d) Cells were transfected with pcDNA-Ins $2^{W T}$ or pcDNA-Ins $2^{\mathrm{C} 96 Y}$. DNA was extracted, electrophoresed in $2 \%$ agarose gel, stained with SYBR Green I, and visualized by UV transillumination. (e) Cells were cotransfected with pEGFP and either pcDNA-Ins2 ${ }^{W T}$ or pcDNA-Ins2 $2^{C 96 Y}$. The transfected cells and apoptotic cells were visualized by GFP fluorescence and annexin V staining, respectively. Original magnification: $\times 200$.

Overexpression of insulin $2^{\mathrm{C} 96 \mathrm{Y}}$ leads to apoptosis in MIN6 cells. Induction of Chop and postnatal hyperglycemia in Ins2 $2^{\mathrm{C} 96 Y}$ mice suggests that the mutant insulin causes ER stress and leads to apoptosis in $\beta$ cells via Chop induction. To test this hypothesis, we overexpressed wild-type and Cys96Tyr mutant insulin 2 in MIN6 cells (Figure 3). We generated fusions of mouse insulin and EGFP for real-time monitoring of apoptosis (Figure 3a). When cells were transfected with the
pIns2 ${ }^{W T}$-EGFP plasmid, fluorescence was observed in transfected cells 24 hours after transfection, and apparently increased with time up to 60 hours (Figure 3a). When cells were transfected with the pIns2 ${ }^{\mathrm{C} 96 Y}$-EGFP plasmid, fluorescent cells were morphologically similar to those expressing $I n s 2^{W T}$-EGFP at 24 hours. However, cells expressing Ins $2^{\mathrm{C} 96 \mathrm{Y}}$-EGFP became round and detached from dishes at 36 hours and thereafter. These results indicate that cells expressing Ins ${ }^{\text {C96Y }}$-EGFP undergo cell death.

To exclude artificial effects of fusion proteins, cells were cotransfected with an EYFP-ER expression plasmid and either the pcDNA-Ins $2^{W T}$ plasmid or the pcDNA-Ins2 ${ }^{C 96 Y}$ plasmid. Cells expressing Ins $2^{W T}$ showed ER-like fluorescence, whereas cells expressing Ins2 ${ }^{\mathrm{C} 96 \mathrm{Y}}$ became round and detached from dishes (Figure 3b). Staining with Hoechst 33258 revealed that cells expressing Ins $2^{\mathrm{C} 96 \mathrm{Y}}$ undergo apoptosis (Figure 3c). Transfection with pcDNA-Ins2 ${ }^{296 Y}$ plasmid led to formation of DNA ladders, which is characteristic of apoptotic cells (Figure 3d). Annexin V staining revealed that cells expressing Ins2 ${ }^{\mathrm{C} 96 \mathrm{Y}}$ undergo apoptosis (Figure $3 e)$. Considering these results together, we conclude that overexpression of Ins $2^{\mathrm{C} 96 \mathrm{Y}}$ causes ER stress and leads to Chop-mediated apoptosis.

Characteristics of double-mutant mice with Ins 2 mutation and Chop disruption. To further investigate the involvement of Chop in ER stress-mediated diabetes, we generated double-mutant mice with mutation in the Ins2 gene and disruption in the Chop gene. Progeny were obtained by crossing Chop ${ }^{-/-}$mice with Ins2 ${ }^{\text {WT/C96Y }}$ mice. Figure $4 \mathrm{a}$ shows typical examples of genotyping. Nine genotypes of mice were generated: Ins $2^{\text {WT } T W T} \mathrm{Chop}^{+/}$, Ins $2^{W T / W T} \mathrm{Chop}^{+/-}$, Ins $2^{\mathrm{WT} / W T} \mathrm{Chop}^{-/,}$, Ins $2^{W T / C 96 Y} \mathrm{Chop}^{+/+}$, Ins2 ${ }^{W T / C 96 Y} \mathrm{Chop}^{+/-}$, Ins2 ${ }^{W T / C 96 Y} \mathrm{Chop}^{-/-}$, Ins2 ${ }^{\mathrm{C} 96 \mathrm{Y} / \mathrm{C} 96 \mathrm{Y}} \mathrm{Chop}^{+/+}$, Ins $2^{\mathrm{C} 96 \mathrm{Y} / \mathrm{C} 96 \mathrm{Chop}} \mathrm{Ch}^{+-}$, and Ins $2^{\mathrm{C} 96 \mathrm{Y} / \mathrm{C} 96 \mathrm{Y}} \mathrm{Chop}^{-/-}$, all of which had the same genetic background (C57BL/6). As shown in Figure 1, Ins $2^{W T / C 96 Y}$ and Ins $2^{C 96 Y / C 96 Y}$ mice developed hyperglycemia by 8 weeks of age. Therefore, we measured body weights, blood glucose levels, and pancreatic insulin content of each genotype at 8 weeks of age (Figure 4b). Ins2 ${ }^{C 96 Y / C 96 Y}$ mice of all Chop genotypes showed loss of body weight, severe hyperglycemia, and reduced pancreatic insulin content. There was no statistically significant difference among $\mathrm{Chop}^{+/+}, \mathrm{Chop}^{+/}$, and $\mathrm{Chop}^{-/-}$mice. Ins $2^{\mathrm{C} 96 \mathrm{Y} / \mathrm{C} 96 \mathrm{Y}}$ mice were often perinatally lethal due to defective insulin secretion in response to glucose (data not shown). In contrast, among the Ins2 $2^{W T / C 96 Y}$ mice, the Chop ${ }^{-/-}$mice did not develop hyperglycemia and had significantly higher body weights and pancreatic insulin content than did $\mathrm{Chop}^{+/-}$and $\mathrm{Chop}^{+/+}$mice. There was little difference in these parameters between $\mathrm{Chop}^{+/-}$and $\mathrm{Chop}^{+/+}$mice. These results are consistent with our previous finding that pancreatic islets from $\mathrm{Chop}^{-/-}$mice are much more resistant to nitric oxide than are those from $\mathrm{Chop}^{+++}$and $\mathrm{Chop}^{+/-}$animals (11). These results indicate that disruption of the Chop gene delays the onset of diabetes in Ins2 ${ }^{W T / C 96 Y}$ mice but not in Ins2 ${ }^{C 96 Y / C 96 Y}$ mice. 
a

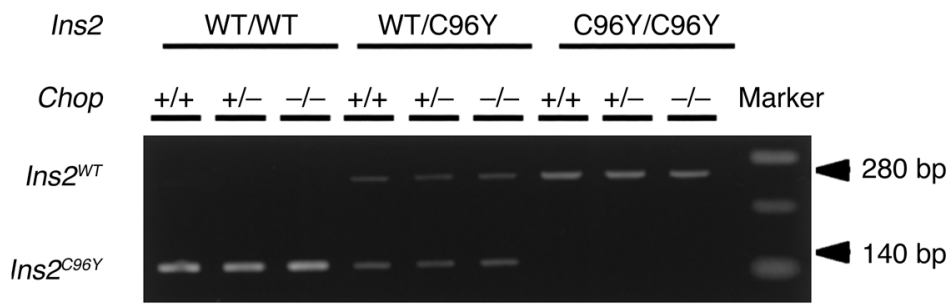

Chop ${ }^{+}$

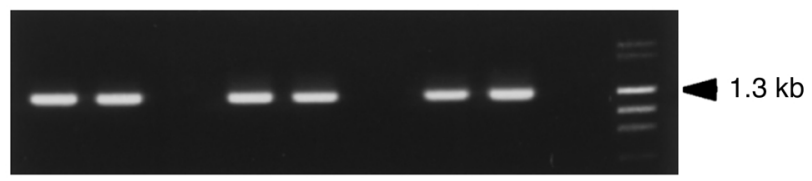

Chop
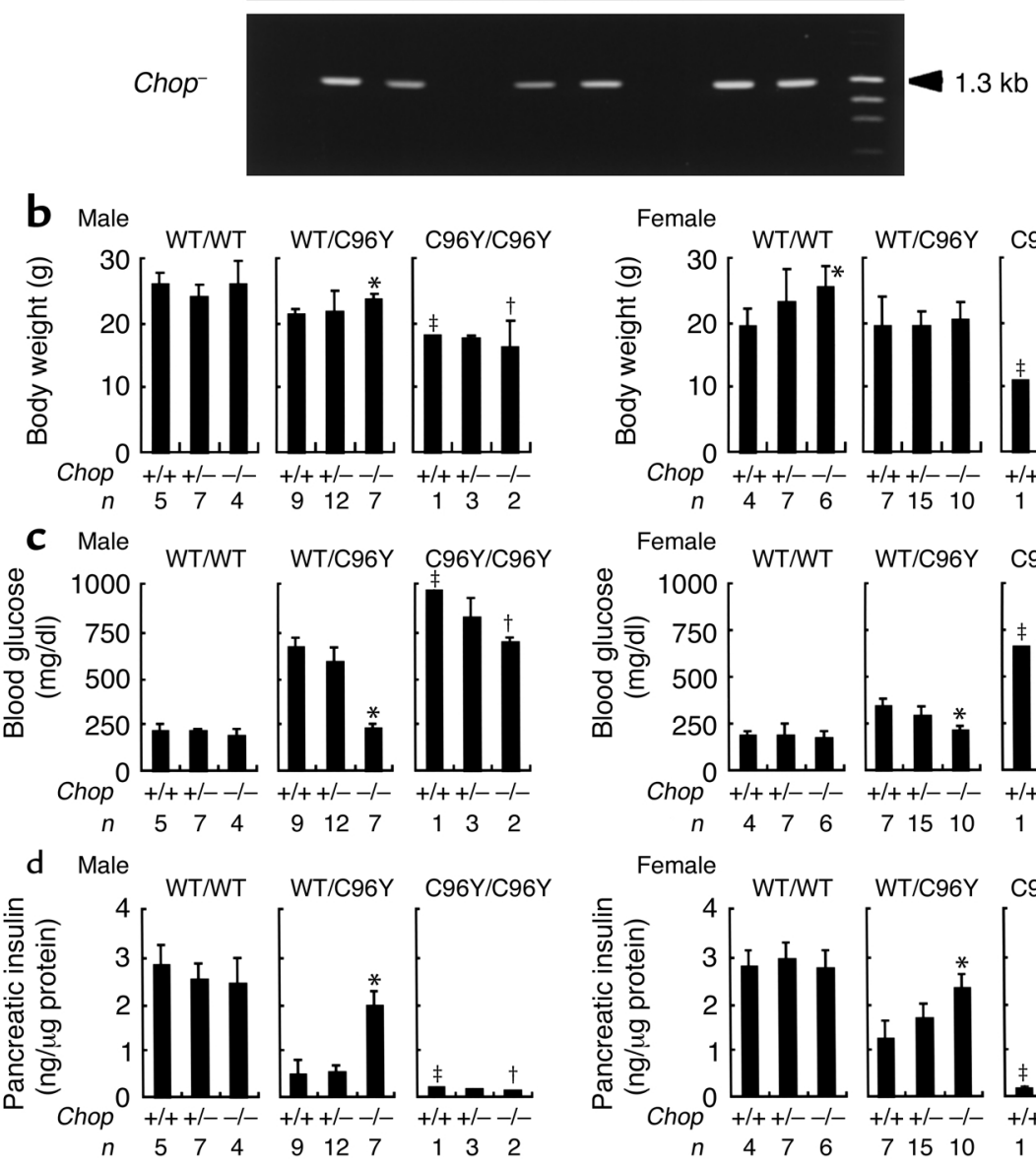
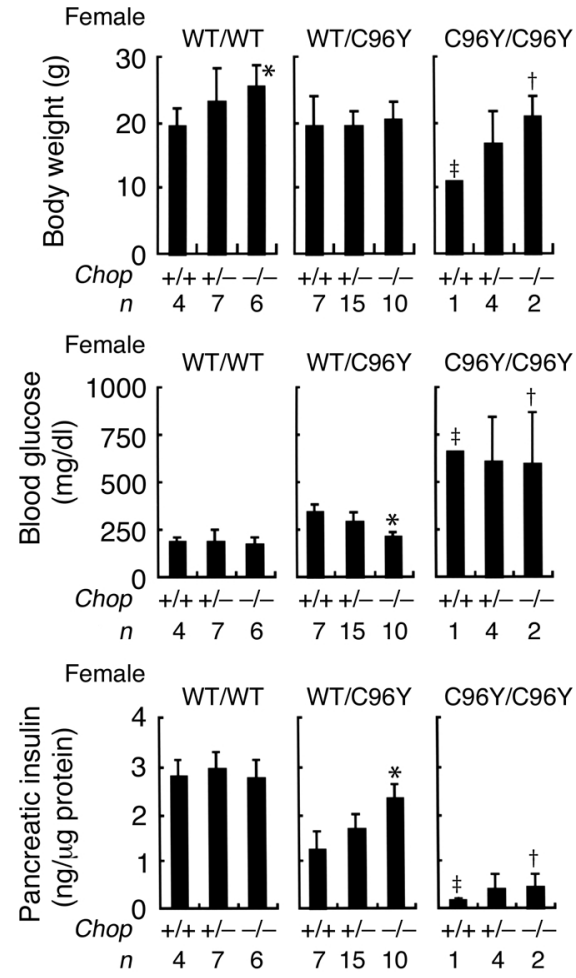

\section{Figure 4}

Characteristics of the double-mutant mice with Ins2 mutation and Chop disruption. (a) Genotyping of doublemutant mice. Representative genotyping of the Ins2 gene by RFLP; the Chop gene from nine mutant lines is shown by PCR. Ins2 exon 3 was amplified by PCR using genomic DNA. The Ins $2^{\mathrm{C} 96 r}$ mutation in Akita mice disrupts an Fnu4HI site in exon 3 of Ins2. Digestion with Fnu4HI did not change the size of the PCR product from the mutated allele (280 bp), but it decreased that of the wild-type allele to $140 \mathrm{bp}$. Primers for wild-type and mutated Chop mice are described in Methods. The left lane shows 100-bp DNA ladder markers (top panel) and $\lambda \mathrm{DNA} /$ Hind III $+\phi \times 174 \mathrm{DNA} /$ Hae III fragment markers (middle and bottom panels). (b-d) Phenotypic characterization of double-mutant mice at 8 weeks of age. (b) Body weight. (c) Morning blood glucose. (d) Pancreatic insulin content. Data are shown as mean \pm SD. Significant differences among the genotype of the $C H O P$ gene were evaluated by the Student $t$ test: ${ }^{*} P<0.001$ versus $\mathrm{Chop}^{+/+}$. $\ddagger_{\text {Ins }} 2^{\mathrm{C} 96 \gamma / \mathrm{C} 96 \mathrm{Cr}} \mathrm{Chop}^{+/+}$mice died within days of birth. Therefore, tdata are shown as only one mouse and $¥$ data are shown as means \pm ranges $(n=2)$.
There was no statistically significant difference in body weight among $\mathrm{Chop}^{+/+}$, Chop ${ }^{+/-}$, and $\mathrm{Chop}^{-/-}$male mice. The female $\mathrm{Chop}^{-/-}$mice were seen to have higher body weight than female $\mathrm{Chop}^{+/+}$animals had. In addition, the development of obesity and fat deposits in the $\mathrm{Chop}^{-/-}$mice after 16 weeks of age appeared to be accelerated (data not shown). It has been suggested that Chop inhibits adipose differentiation by interfering with the accumulation of adipogenic C/EBP isoforms $(17,18)$. Therefore, we speculate that targeted disruption of the Chop gene results in obesity by increasing adipocyte differentiation. In this study, the Chop ${ }^{-1}$ mice, generated on a C57BL/ 6 strain, were backcrossed only two generations from the original $129 / \mathrm{Sv} \times$ $\mathrm{C} 57 \mathrm{BL} / 6$ mice. Therefore, it is also possible that the $129 /$ Sv background influences body weight.
Disruption of the Chop gene protects islet cells of Ins $2^{W T / C 96 Y}$ mice from apoptosis. The effect of disruption of the Chop gene in apoptosis of $\beta$ cells in Ins $2^{\text {WT/C96Y }}$ mice was examined histologically (Figure 5). At 4 weeks of age, Chop induction was observed in Ins $2^{W T / C 96 Y} \mathrm{Chop}^{+/+}$islets. Distribution of Chop-positive cells was similar to that of insulin-positive cells, suggesting that Chop-positive cells are $\beta$ cells. In Ins $2^{\text {WT/C96Y }}$ mice, the size of islets decreased markedly, and the mass of insulin-positive cells diminished. Apoptotic (TUNEL-positive) cells were clearly

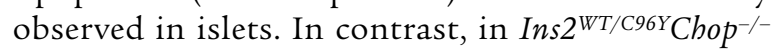
mice, islet size was fairly well retained, and the number of TUNEL-positive cells was much smaller. The number of TUNEL-positive cells per islet in Ins ${ }^{W T / C 96 Y} \mathrm{Chop}^{+/+}$mice was $10.50 \pm 10.73(n=20)$, 
$\mathbf{a}$

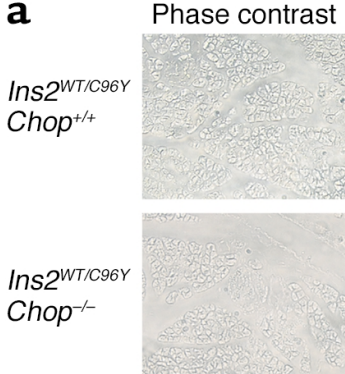

b
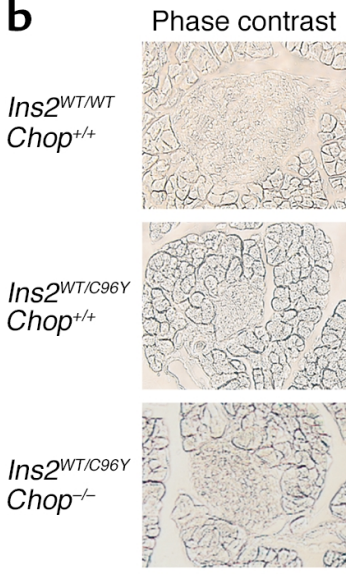

Insulin
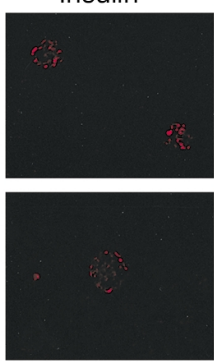

Insulin
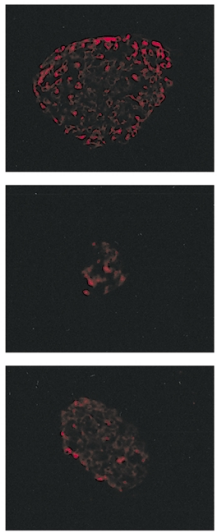

Chop
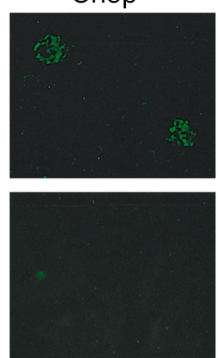

TUNEL
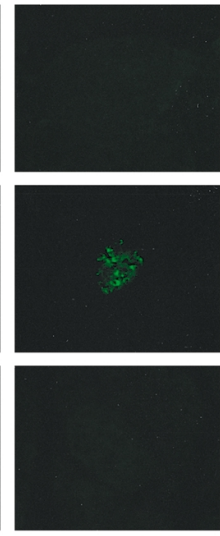

\section{Figure 5}

Immunohistochemical detection of Chop and apoptosis in islets of male Ins $2^{W T / W T} \mathrm{Chop}^{+/+}$, Ins $2^{W T / C 96 Y} \mathrm{Chop}^{+/+}$, and Ins ${ }^{W T / C 96 r} \mathrm{Chop}^{-/-}$mice. (a) Immunostaining of insulin and Chop. The pancreata from Ins $2^{W T / C 96 Y} \mathrm{Chop}^{+/+}$and Ins2 ${ }^{W T / C 96 Y} \mathrm{Chop}^{-/-}$male mice at 4 weeks of age were fixed and then costained for insulin (red) and Chop (green). Phase-contrast images and fluorescence images of the same fields are shown. Original magnification: $\times 100$. (b) Apoptosis detection by the TUNEL method. The pancreata from Ins $2^{W T / W T} \mathrm{Chop}^{+/+}$, Ins2 ${ }^{W T / C 96 Y} \mathrm{Chop}^{+/+}$, and Ins $2^{W T /{ }^{W 6 r}} \mathrm{Chop}^{-/-}$male mice were obtained and costained for insulin (red) and TUNEL-positive cells (green). Phase-contrast images and fluorescence images of the same fields are shown. Original magnification: $\times 200$.

whereas that in Ins2 ${ }^{\text {WT }} /{ }^{C 96 Y} \mathrm{Chop}^{-/-}$mice was $1.10 \pm 1.74$ $(n=20)$. In Ins $2^{\mathrm{C} 96 Y / C 96 Y}$ mice, disruption of the Chop gene failed to protect islets from apoptosis (data not shown). These data indicate that the hyperglycemia in Ins $2^{\mathrm{C} 96 \mathrm{Y}}$ mutant mice was primarily due to a specific decrease of $\beta$ cells through ER stress-mediated apoptosis, and that disruption of the Chop gene rescues $\beta$ cells of Ins $2^{W T / C 96 Y}$ mice from apoptosis.

Disruption of the Chop gene delays the onset of diabetes in Ins $2^{W T / C 96 Y}$ mice. To determine whether disruption of the Chop gene would block or delay the onset of diabetes in Ins $2^{W T / C 96 Y}$ mice, we monitored blood glucose levels in Ins2 ${ }^{W T / C 96 Y} \mathrm{Chop}^{+/+}$, Ins $2^{\text {WT/C96Y }} \mathrm{Chop}^{+/-}$, and Ins2 ${ }^{W T / C 96 Y} \mathrm{Chop}^{-/-}$mice (Figure 6). Both Ins $2^{W T / C 96 Y} \mathrm{Chop}^{+/+}$and Ins2 ${ }^{W T / C 96 Y} \mathrm{Chop}^{+/-}$mice developed hyperglycemia beginning at 4 weeks of age. Blood glucose levels in these mice increased up to about 800 $\mathrm{mg} / \mathrm{dl}$, at which point mice were 20 weeks of age. In contrast, blood glucose in Ins $2^{W T / C 96 Y} \mathrm{Chop}^{-/-}$mice remained unchanged (less than $250 \mathrm{mg} / \mathrm{dl}$ ) until the mice reached 8 weeks of age. At that point levels began

to increase; at 20 weeks the blood glucose level in these mice was about $500 \mathrm{mg} / \mathrm{dl}$. Thus, disruption of the Chop gene delayed the onset of diabetes in Ins $2^{W T / C 96 Y}$ mice by $8-10$ weeks; hyperglycemia was somewhat overcome but was not prevented.

\section{Discussion}

We obtained evidence that mutation in Akita mice causes ER stress, induces Chop mRNA, and leads to the apoptosis of $\beta$ cells. Targeted disruption of the Chop gene significantly prevented Ins2 ${ }^{C 96 Y}$-induced diabetes by decreasing ER stress-mediated apoptosis in $\beta$ cells. Therefore, perturbation in functions of the ER in $\beta$ cells leads to diabetes through Chop induction.

The ER has a mechanism to monitor protein folding called "quality control" (19). In Ins $2^{C 96 Y}$ mutant mice, the mutant insulin is not secreted; it is trapped in the ER and is intracellularly degraded (14). This mouse has two nonallelic insulin genes (Ins1 and Ins2). In wild-type mice, Ins 2 transcripts represent the majority of total insulin. Although Ins $2^{C 96 Y}$ mice develop severe diabetes, single-mutant mice that are either Ins $1^{-/-}$or Ins2-/- do not, and plasma insulin levels in these mice are normal (20). Hence, we speculated that diabetes in Ins $2^{\mathrm{C} 96 Y}$ mice is induced by $\beta$ cell disruption mediated by ER stress, rather than by the loss of active insulin. This notion is supported by evidence of apoptosis and the induction of Chop mRNA in the pancreas of Ins $2^{\mathrm{C} 96 Y}$ mutant mice. In Ins $2^{-/-}$mutant mice, to overcome insulin deficiency, the $\beta$ cell mass is increased and synthesis of insulin 1 is enhanced (20). In Ins ${ }^{2 C 96 Y}$ mice, on the other hand, expression of the insulin gene is upregulated to compensate for insulin deficiency, but synthesized mutant insulin induces ER stress, $\beta$ cell death follows, and glucose metabolism is decreased (Figure 2).

$\beta$ cells are strongly engaged in protein secretion and have highly developed ER. ER stress transducer proteins, including Ire $1 \alpha$ and PERK, are expressed at high

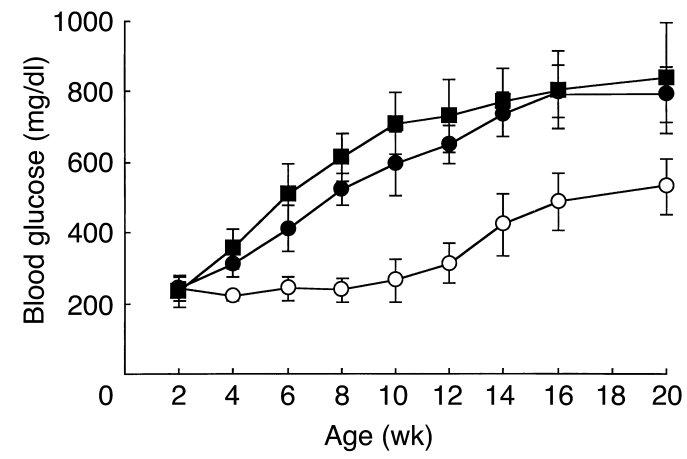

Figure 6

Development of diabetes in male Ins $2^{W T / C 96 r} \mathrm{Chop}^{+/+}$,

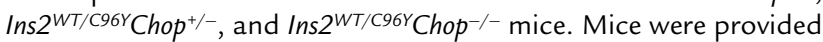
food ad libitum, and blood glucose was measured between 9:00 a.m. and 10:00 a.m. Ins2 ${ }^{W T / C 96 Y} \mathrm{Chop}^{+/+}$mice (filled squares), Ins ${ }^{W T / C 96 Y} \mathrm{Chop}^{+/-}$mice (filled circles), and Ins $2^{W T / C 96 Y} \mathrm{Chop}^{-/-}$mice (open circles) are represented. Data are shown as mean $\pm \operatorname{SD}(n=7)$. 
levels in $\beta$ cells. This high expression may be necessary for strict quality control of secretory proteins in $\beta$ cells, and disturbance of protein traffic in ER may lead to cell death. Two recent studies using genetically engineered mice revealed that PERK disruption and defective eIF $2 \alpha$ phosphorylation results in $\beta$ cell insufficiency, thereby demonstrating that the PERK-eIF $2 \alpha$ pathway plays an important role in translational control in ER stress $(9,10)$. Postnatally increased apoptosis in $\beta$ cells was observed in $P E R K^{-/-}$mice. Electron micrographs of islets in $\mathrm{PERK}^{-/-}$mice revealed dilated $E R$ in $\beta$ cells, and a reduced number and size of secretory granules. Similar changes are observed in islets of Akita mice (14). Mutations in the human PERK gene cause Wolcott-Rallison syndrome, an autosomal recessive disease with severe diabetes that develops in infancy (21). Patients have pancreatic hypoplasia and a reduced number of $\beta$ cells (22). Taken together, these results suggest that chronic ER stress results in hyperglycemia due to reduction in $\beta$ cell mass, and that $\beta$ cells are most susceptible to ER stress.

In contrast to patients with type 1 diabetes, those with type 2 diabetes are often hyperinsulinemic during the prediabetic or early diabetic phase. Overload of pancreatic $\beta$ cells under conditions such as hyperglycemia, obesity, and long-term treatment with sulfonylureas is often observed in type 2 diabetes. This increased demand of insulin may exhaust the secretory capacity of $\beta$ cells. Indeed, when MIN6 cells were transfected with wild-type insulin, highly overexpressed cells were prone to apoptosis (data not shown). These findings support the idea that chronic ER stress caused by excessive demand of insulin is responsible for $\beta$ cell exhaustion. However, disruption of the Chop gene failed to prevent diabetes in Ins $2^{W T / C 96 Y}$ mice, although it did delay the onset of disease. In the case of Ins2 ${ }^{C 96 Y / C 96 Y}$ mice, disruption of the Chop gene could not delay the onset of diabetes. Chop induction in response to ER stress is highly dependent on PERK (23). In islets of $P E R K^{-1-}$ mice, induction of Chop is attenuated, but $\beta$ cells undergo apoptosis (9). Thus, the involvement of other death pathways, such as activation of JNK via Ire1 $\alpha(24)$ and activation of caspase-12 (8), seems likely.

There are similarities in development between type 1 and type 2 diabetes. Once mild hyperglycemia occurs, a common pathway of diabetes leads to further severe hyperglycemia. Our results provide evidence suggesting that the ER in $\beta$ cells modifies the development of diabetes. We propose that overload of $\beta$ cells causes chronic ER stress and leads to apoptosis of $\beta$ cells via Chop induction. Therefore, targeted suppression of Chop expression or its function in $\beta$ cells may be a new therapeutic approach for preventing the onset of both type 1 and type 2 diabetes.

\section{Acknowledgments}

We thank J. Miyazaki (Osaka University) for providing MIN6 cells, R. Shindo (this laboratory) for technical assistance, and N.J. Hoogenraad (La Trobe University) and M. Ohara (Fukuoka, Japan) for comments on the manuscript. This work was supported in part by a Grant-in-Aid from the Ministry of Education, Science, Sports and Culture of Japan.

1. Stefan, Y., et al. 1982. Quantitation of endocrine cell content in the pancreas of nondiabetic and diabetic humans. Diabetes. 31:694-700.

2. Rahier, J., Goebbels, R.M., and Henquin, J.C. 1983. Cellular composition of the human diabetic pancreas. Diabetologia. 24:366-371.

3. Kaufman, R.J. 1999. Stress signaling from the lumen of the endoplasmic reticulum: coordination of gene transcriptional and translational controls. Genes Dev. 13:1211-1233.

4. Mori, K. 2000. Tripartite management of unfolded proteins in the endoplasmic reticulum. Cell. 101:451-454.

5. Travers, K.J., et al. 2000. Functional and genomic analyses reveal an essential coordination between the unfolded protein response and ER-associated degradation. Cell. 101:249-258.

6. Barone, M.V., Crozat, A., Tabaee, A., Philipson, L., and Ron, D. 1994. Chop (GADD153) and its oncogenic variant, TLS-Chop, have opposing effects on the induction of G1/S arrest. Genes Dev. 8:453-464.

7. Matsumoto, M., Minami, M., Takeda, K., Sakao, Y., and Akira, S. 1996. Ectopic expression of Chop (GADD153) induces apoptosis in M1 myeloblastic leukemia cells. FEBS Lett. 395:143-147.

8. Nakagawa, T., et al. 2000. Caspase-12 mediates endoplasmic-reticulumspecific apoptosis and cytotoxicity by amyloid-b. Nature. 403:98-103.

9. Harding, H.P., et al. 2001. Diabetes mellitus and exocrine pancreatic dysfunction in perk $/-$ mice reveals a role for translational control in secretory cell survival. Mol. Cell. 7:1153-1163.

10. Scheuner, D., et al. 2001. Translational control is required for the unfolded protein response and in vivo glucose homeostasis. Mol. Cell. 7:1165-1176.

11. Oyadomari, S., et al. 2001. Nitric oxide-induced apoptosis in pancreatic $\beta$ cells is mediated by the endoplasmic reticulum stress pathway. Proc. Natl. Acad. Sci. USA. 98:10845-10850.

12. Kayo, T., and Koizumi, A. 1998. Mapping of murine diabetogenic gene Mody on chromosome 7 at D7Mit258 and its involvement in pancreatic islet and $\beta$ cell development during the perinatal period. J. Clin. Invest. 101:2112-2118.

13. Yoshioka, M., Kayo, T., Ikeda, T., and Koizumi, A. 1997. A novel locus, Mody4, distal to D7Mit189 on chromosome 7 determines early-onset NIDDM in nonobese C57BL/6 (Akita) mutant mice. Diabetes. 46:887-894.

14. Wang, J., et al. 1999. A mutation in the insulin 2 gene induces diabetes with severe pancreatic $\beta$-cell dysfunction in the Mody mouse. J. Clin. Invest. 103:27-37.

15. Oyadomari, S., et al. 2001. Coinduction of endothelial nitric oxide synthase and arginine recycling enzymes in aorta of diabetic rats. Nitric Oxide. 5:252-260.

16. Davalli, A.M. 1995. Function, mass, and replication of porcine and rat islets transplanted into diabetic nude mice. Diabetes. 44:104-111.

17. Tang, Q.Q., and Lane, M.D. 2000. Role of C/EBP homologous protein (Chop-10) in the programmed activation of CCAAT/enhancer-binding protein-b during adipogenesis. Proc. Natl. Acad. Sci. USA. 97:12446-12450.

18. Batchvarova, N., Wang, X.Z., and Ron, D. 1995. Inhibition of adipogenesis by the stress-induced protein Chop (Gadd153). EMBOJ. 14:4654-4661.

19. Kopito, R.R. 1997. ER quality control: the cytoplasmic connection. Cell. 88:427-430.

20. Leroux, L., et al. 2001. Compensatory responses in mice carrying a null mutation for Ins1 or Ins2. Diabetes. 50(Suppl. 1):S150-S153.

21. Delepine, M., et al. 2000. EIF2AK3, encoding translation initiation factor 2 -a kinase 3 , is mutated in patients with Wolcott-Rallison syndrome. Nat. Genet. 25:406-409.

22. Thornton, C.M., Carson, D.J., and Stewart, F.J. 1997. Autopsy findings in the Wolcott-Rallison syndrome. Pediatr. Pathol. Lab. Med. 17:487-496.

23. Harding, H.P., et al. 2000. Regulated translation initiation controls stressinduced gene expression in mammalian cells. Mol. Cell. 6:1099-1108.

24. Urano, F., et al. 2000. Coupling of stress in the ER to activation of JNK protein kinases by transmembrane protein kinase IRE1. Science. 287:664-666. 